\title{
Cardioprotective natural compound pinocembrin prevents acute ischemic myocardial injury through enhancing glycolysis
}

\author{
Jingrong Lin ${ }^{1}$, Yanjun Zheng², Guoqing $\mathrm{Wan}^{2}$, and Xuefeng $\mathrm{Gu}^{2}$ \\ ${ }^{1}$ Shanghai Institute of Hypertension \\ ${ }^{2}$ Shanghai University of Medicine \& Health Sciences Affiliated Zhoupu Hospital
}

May 21, 2020

\begin{abstract}
Background and Purpose: Emerging evidence has shown that pinocembrin protects myocardial from ischemic injury in animals. However, it is unknown whether it has cardioprotection when given at the onset of reperfusion. Also, mechanisms mediating the cardioprotective actions of pinocembrin were largely unknown. Thus, this study aimed to investigate the effects of pinocembrin postconditioning on ischemia-reperfusion (I/R) injury and the underlying mechanisms. Experimental Approach: In vivo mouse model of myocardial I/R injury, ex vivo isolated rat heart with global I/R and in vitro hypoxia/reoxygenation (H/R) injury model for primary cardiomyocytes were used. Key Results: We found that pinocembrin postconditioning significantly reduced the infarct size and improved cardiac contractile function after acute myocardial I/R. Mechanically, in primary cardiomyocytes we found that pinocembrin may confer protection in part via direct stimulation of cardiac glycolysis via promoting the expression of glycolytic enzyme, PFKFB3. Besides, PFKFB3 inhibition abolished pinocembrin-induced glycolysis and protection in cardiomyocytes. More importantly, PFKFB3 knockdown via cardiotropic adeno-associated virus (AAV) abrogated cardioprotective effects of pinocembrin. Conclusions and Implications: In conclusion, these results established that the acute cardioprotective benefits of pinocembrin are mediated in part via glycolytic stimulation through PFKFB3, which may provide a new therapeutic target to impede the progression of myocardial I/R injury.
\end{abstract}

Abbreviations: I/R: ischemia-reperfusion; H/R, hypoxia/reoxygenation; PFKFB3, 6-phosphofructo-2kinase/fructose-2, 6-bisphosphatase isoform 3; AAV; adeno-associated virus

What is already known:

Pinocembrin protects myocardial from ischemic injury in animals.

What this study adds:

Pinocembrin postconditioning significantly reduced the infarct size and improved cardiac contractile function after acute myocardial I/R.

Acute cardioprotective benefits of pinocembrin are mediated in part via glycolytic stimulation through PFKFB3.

What is the clinical significance:

Pinocembrin may be an effective agent for improving tissue perfusion in I/R-related diseases.

\section{Introduction}


Cardiovascular disease remains the leading cause of death worldwide (Bice \& Baxter, 2015). Currently, there are few effective drugs to protect the heart after ischemia/reperfusion (I/R) injury. Therefore, it has become a hot research field to find the molecular mechanism of coronary artery disease progression and development that can protect myocardium from I/R injury. With this in mind, more and more attention is focused on pharmacological interventions because it can induce cardioprotection and easy to implement (Zheng et al., 2017). However, for patients with ischemic heart disease, there are still many restrictive drugs available clinically.

Traditional Chinese medicine has a history of thousands of years and provides a large amount of medicinal materials. Pinomline (5,7-dihydroxyflavone) is an abundant flavonoid isolated from propolis and some plants. It has various biological functions such as anti-inflammatory, anti-oxidation, anti-bacterial, etc. (Hanieh et al., 2017). Previous studies have confirmed that pinocembrin confer neuroprotective effects during cerebral ischemic I/R (Saad, Abdel Salam, Kenawy, \& Attia, 2015; Shi et al., 2011; Tao, Shen, Sun, Chen, \& Yan, 2018). Recent research has also tried to determine whether pinocembrin is beneficial for cardiac injury. For example, several studies suggest that pinocembrin can improve the cardiac function of myocardial I/R rats, reduce ventricular arrhythmia, and reduce the area of myocardial infarction (Lungkaphin et al., 2015; Zhang, Xu, Hu, Yu, \& Bai, 2018). However, it is still unclear whether pinocembrin given at the onset of reperfusion has cardioprotective effects, which is a more clinically effective method. In addition, the underlying mechanism by which pinocembrin can provide cardioprotection is largely unknown.

Recent studies have confirmed that there are changes in energy metabolism in many human diseases, and targeted energy metabolism may have therapeutic potential on these diseases (Gohil et al., 2010). Due to the mechanical function of the heart, it is an organ with high energy requirements. In general, most of the energy (about 70\%) of a healthy heart comes from the $\beta$-oxidation of fatty acids, and the rest of the energy comes from glucose oxidation (Lopaschuk, Ussher, Folmes, Jaswal, \& Stanley, 2010). Nevertheless, in a pathological environment, substrate utilization may change (Lionetti, Stanley, \& Recchia, 2011). In patients with diabetes, circulating blood glucose levels at admission are related to the clinical outcome after acute myocardial infarction (AMI), suggesting that it may be related to myocardial metabolism (Malmberg, Norhammar, Wedel, \& Ryden, 1999). Metabolic shift from $\beta$-oxidation to glycolysis metabolism will reduce the cell's need for oxygen by 11-13\%, and NAD+ precursors have been shown to activate cellular glycolysis to protect the heart from ischemic injury (Nadtochiy, Wang, Nehrke, Munger, \& Brookes, 2018). It is worth noting that redirect energy metabolism to glycolysis can reduce oxidative damage and inhibit apoptosis (Hunter, Hendrikse, \& Renan, 2007; Jeong, Kim, Cho, \& Kim, 2004; Vaughn \& Deshmukh, 2008). However, few agents that target energy metabolism are clinically safe and useful for patients. At the same time, it is unclear whether and how pinocembrin regulates acute myocardial I/R glycolysis.

To address these issues, this study was designed to (i) the current study was designed to determine the role of pinocembrin in rat and mouse cardiac I/R injury ex vivo and in vivo respectively. (ii) clarify the effects of pinocembrin on the glycolytic metabolism during I/R; (iii) explore the underlying molecular basis that contributes to the pinocembrin-induced cardioprotection. Our results provide new insight into the mechanism of pinocembrin-induced cardioprotection and suggest a potential application value of pinocembrin in the protection of hearts against I/R injury.

\section{Methods}

\subsection{Chemicals and reagents}

Pinocembrin were obtained from Sigma-Aldrich (St. Louis, MO, USA). 3-(3-Pyridinyl)-1-(4-pyridinyl)-2propen-1-one (3PO) was from EMD Millipore (MA, USA). All other reagents were from Sigma-Aldrich (St. Louis, MO, USA).

\subsection{Animals}

250-300 g Adult Male Sprague-Dawley (SD) rats and C57BL/6 mice (age, 8-10 weeks) from Shanghai Slac Laboratory Animal Co. Ltd. were cared in accordance with the Guidelines for Care and Use of Laboratory 
Animals published by the US National Institutes of Health (NIH Publication, 8th Edition, 2011). Animal procedures were approved by the Institutional Review Board of Shanghai University of Health and Medicine (Shanghai, China).

$2.3 \mathrm{I} / \mathrm{R}$ injury model in Langendorff-perfused rat hearts

As mentioned before, the heart was rapidly excised at $37{ }^{\circ} \mathrm{C}$ and perfused with Krebs-Henseleit buffer (KHB) at a constant pressure of $80 \mathrm{mmHg}$ using Langendorff technique (Xie et al., 2005). Left ventricular $(\mathrm{LV})$ pressure was monitored with a water-filled latex balloon connected to a pressure sensor (AD instrument, Bella Vista, New South Wales, Australia) and inserted into the left ventricle cavity to achieve left ventricular end-diastolic pressure (LVEDP) at 0 to $10 \mathrm{~mm} \mathrm{Hg}$. PowerLab system (AD instrument, Australia) was used to monitor left ventricular imaging pressure (LVDP), left ventricular imaging pressure (LVEDP), maximum rate of pressure changes over time $(+\mathrm{dP} / \mathrm{dtmax})$ and pressure decay over time $(-\mathrm{dP} / \mathrm{dtmax})$. Pinocembrin's final concentration of 10,30 and $100 \mu \mathrm{M}$ perfusate was added with 5 minutes of reperfusion. In order to assess the infarct size, the isolated rat heart was re-perfused for 2 hours after 30 minutes of ischemia. The slices were incubated in $1 \% \mathrm{w} / \mathrm{v}$ triphenyltetrazolium chloride (TTC, $\mathrm{pH} 7.4$ ) for $15 \mathrm{~min}$, and then fixed in $10 \%$ formaldehyde. Image-Pro-Plus software (media cybernetics) was used to calculate the infarct area. The infarct area was expressed as a percentage of the LV area at risk.

\subsection{Myocardial I/R Model}

Surgical ligation of the left coronary artery (LCA) was performed as described previously (Qin et al., 2017). Briefly, mice were anesthetized with ketamine $(50 \mathrm{mg} / \mathrm{kg})$ and pentobarbital sodium $(50 \mathrm{mg} / \mathrm{kg})$ by intraperitoneal injection, followed by orally intubated and ventilated. The core body temperature is always maintained at $37^{\circ} \mathrm{C}$. An internal sternotomy was then performed with electrocautery, and then the proximal LCA was displayed and ligated. After 30 minutes of coronary artery occlusion, the suture was cut and the blood vessels were allowed to reperfusion. After 24 hours of reperfusion, the mice were anesthetized with isoflurane. Transthoracic echocardiography was used to determine the left ventricular ejection fraction. After reperfusion, blood samples were collected and centrifuged at $3000 \mathrm{rpm}$ for 10 minutes for cardiac troponin- $\mathrm{T}$ $(\mathrm{cTnT})$ and lactate dehydrogenase (LDH) measurements. Measurement of area at risk and infarct size was performed as reported previously. Each of the myocardial slices were weighed and the areas of infarction, risk, and nonischemic left ventricle were assessed by a blinded observer using computer-assisted planimetry (NIH ImageJ 1.37).

\subsection{Isolation and culture of primary cardiomyocytes}

Continuous enzymatic digestion and isolation were used to obtain neonatal rat and mouse cardiomyocytes (Irvine et al., 2013; Irvine et al., 2012). Neonatal rats were decapitated and hearts were immediately placed in HBSS. The ventricle was taken and digested with trypsin overnight at $4{ }^{\circ} \mathrm{C}$ and collagenase four times at $37^{\circ} \mathrm{C}$ for $10 \mathrm{~min}$. Cardiomyocytes were suspended in sterile DMEM, supplemented with penicillin $100 \mathrm{Uml}^{-1}$, streptomycin $100 \mathrm{mgml}^{-1}$ and $10 \%$ fetal bovine serum. The cells were pre-plated twice $\left(37^{\circ} \mathrm{C}\right.$ for 45 minutes) to reduce fibroblast contamination. Hypoxia/reoxygenation $(\mathrm{H} / \mathrm{R}$, which simulated $\mathrm{MI} / \mathrm{R}$ in vivo ) was employed as previously described (Hou et al., 2019).

\subsection{Measurement of LDH release and cTnT release}

Necrotic cell death was evaluated by supernatant LDH activity, as in previous studies (Kishi et al., 2015). A spectrophotometric kit (Nanjing jiancheng, Jiangsu, China) was used according to the manufacturer's instruction. In short, $20 \mu \mathrm{l}$ supernatants were collected in a 96 -well, then $25 \mu \mathrm{l}$ matrix buffer and $5 \mu$ l coenzyme I was added to the 96 -well. The mixture was incubated at $37^{\circ} \mathrm{C}$ for $15 \mathrm{~min}$. After $25 \mu \mathrm{l} 2,4$-dinitrophenylhydrazine was added to each well and incubated at $37^{\circ} \mathrm{C}$ for $15 \mathrm{~min}, 250 \mu \mathrm{l} 0.4 \mathrm{M} \mathrm{NaOH}$ was added to each well and the mixture was incubated at room temperature for $5 \mathrm{~min}$. The absorbance value was measured at $450 \mathrm{~nm}$ with the spectrophotometer (BioTek, VT, USA) and the LDH activity was calculated. Plasma cTnT levels as an indicator of cardiomyocyte damage was measured using a mouse cTnT ELISA kit (Elabscience Biotechnology Co., Ltd, Wuhan, China) according to the manufacturer's instruction. 


\subsection{Quantitative real-time PCR}

We isolated total RNA was from the heart tissue with Trizol Reagent (Invitrogen, Carlsbad, CA, USA). Relative quantitation by real-time PCR involved SYBR Green detection of PCR products in real time with the ABI PRISM 7700 Sequence Detection System (Applied Biosystems). The primers were list in Supplemental Table 1. The reactions were conducted in triplicate by heating the reactant to $95{ }^{\circ} \mathrm{C}$ for 5 min, followed by 40 cycles of $94{ }^{\circ} \mathrm{C}$ for $30 \mathrm{~s}, 58{ }^{\circ} \mathrm{C}$ for $30 \mathrm{~s}$ and $72{ }^{\circ} \mathrm{C}$ for $30 \mathrm{~s}$.

2.8 Western blot analysis

Left ventricles were homogenized and the cells were lysed as described previously (Matsushima et al., 2013). The samples were analyzed by SDS-PAGE. Transfer the protein to a polyvinylidene fluoride microporous membrane (Bio Rad) with primary antibody PFKFB3 (Abcam, USA; 1: 1000) and anti-GAPDH (internal control; Kangcheng Co., Ltd., Shanghai, China; 1: 8000). The secondary antibody was coupled to horseradish peroxidase (Cell Signaling Technology, 1: 6000). Enhanced ECL detection kit (Amersham Pharmacia Biotech) was employed to visualize the immunoreaction, followed by exposing to film and quantified with a video documentation system (Gel Doc 2000, Bio-Rad).

\subsection{Generation and administration of adeno-associated virus (AAV)}

Serotype 9 AAV vectors (AAV9) encoding shNC or shPFKFB3 (AAV9-shNC and AAV9-shPFKFB3) were prepared as previously described (Xie et al., 2016). $3 \times 10^{11}$ vg of AAV9-shNC or AAV9-shPFKFB3 were injected intravenously into tail veins as previously described (Somanathan et al., 2014) of 4-5 weeks old male C57 mice. Sham or myocardial ischemia surgeries were conducted 4 weeks after AAV9 injection.

\subsection{Seahorse extracellular flux analyzer assays}

Cellular bioenergetics was measured using a Seahorse XFe24 extracellular flux analyzer in intact cardiomyocytes. We conducted glycolysis stress testing according to the manufacturer's instructions previously reported (He et al., 2017). Glycolysis stress test: Cells were incubated in glucose-free Seahorse assay media supplemented with $1 \mathrm{mM}$ pyruvate at $37^{\circ} \mathrm{C}$ in incubator without $\mathrm{CO} 2$ for 1 -h prior to the assay. Injectors were loaded to add $20 \mathrm{mM}$ Glucose, $1 \mu \mathrm{M}$ Oligomycin and 100mM 2 Deoxy-Glucose (2-DG) and glycolysis, glycolytic capacity and glycolytic reserves were calculated as extracellular acidification rate (ECAR).

\subsection{Statistical analysis}

Data were presented as means \pm SEM. The unpaired, 2-tailed $t$ test was used for comparisons between 2 groups. For multiple comparisons, ANOVA or repeated ANOVA followed by the LSD post hoc test was used

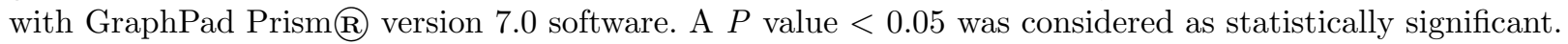

\section{Results}

3.1 Compound pinocembrin significantly improved the cardiac function and reduced infarct size after I/R $e x$ vivo

We perfused isolated rat hearts to explore the cardioprotective effects of pinocembrin against I/R injury, 10 to $100 \mu \mathrm{M}$ pinocembrin were delivered during the first $5 \mathrm{~min}$ of reperfusion (fig 1A). During 45 min reperfusion following 30min ischemia, the contractile function of left ventricular (LV), including LV developed pressure (LVDP), LV end-diastolic pressure (LVEDP), maximal rates of pressure development over time $(+\mathrm{dP} / \mathrm{dtmax})$ and pressure decay over time (-dP/dtmax) were significantly suppressed (fig $2 \mathrm{~A}-\mathrm{D}$ ). Pinocembrin itself does not affect the heart rate during reperfusion (Supplementary fig 1), while it dose-dependently improved the postischemic myocardial performance from 10 to $100 \mu \mathrm{M}$ (fig $2 \mathrm{~A}-\mathrm{D}$ ).

Next, we explore whether pinocembrin improves cell survival during I/R via examining lactate dehydrogenase (LDH) release, an indicator of myocardial injury. Little LDH release was detected in the coronary efflux before ischemia, while LDH release was obviously induced at the end of reperfusion, while pinocembrin significantly inhibited the release of $\mathrm{LDH}$ from 10 to $100 \mu \mathrm{M}$ (fig 2E). Consistently, the I/R-induced infarction after 2 
$\mathrm{h}$ of reperfusion was significantly attenuated by pinocembrin at the concentration of $30 \mu \mathrm{M}$ (fig $2 \mathrm{~F}$ ). These data demonstrated that pinocembrin exhibits beneficial effects on cardiac I/R injury ex vivo .

Compound pinocembrin protects hearts from myocardial I/R injuryin vivo

To explore the cardioprotective effects of pinocembrin on myocardial $\mathrm{I} / \mathrm{R}$ injury in vivo, pinocembrin was intravenously injected into wild-type (WT) mice 5 minutes before the end of sustained ischemia (i.v., 5 $\mathrm{mg} / \mathrm{kg}$ and $10 \mathrm{mg} / \mathrm{kg}$ ), followed by reperfusion for 24 hours (fig 1B). Evans-blue/TTC dye method was used to determine the infarct size. After reperfusion for 24 hours, there was no difference of area at risk (AAR) between each group (Figure 3A and 3B). Nevertheless, compared with I/R group, pinocembrin i.v. treatment significantly reduced the infarct size by $20 \%$ (fig $3 \mathrm{~A}$ and $3 \mathrm{C}$ ). Besides, plasma levels of cTnI and LDH activity were markedly elevated during myocardial I/R, which were both suppressed with pinocembrin i.v. treatment (fig 3 D-E). Furthermore, the echocardiographic results showed that pinocembrin can significantly improve I/R-suppressed the ejection fraction (EF\%) and fractional shortening (FS\%) (fig 3F).

3.3 Protective actions of pinocembrin against cardiomyocyte injury responses in vitro

Next, we examined the direct effects of pinocembrin on cardiomyocytes. To determine whether pinocembrin confers cardioprotective effects through its direct action on the cardiomyocytes, we subjected the isolated neonatal rat and mouse cardiomyocytes to $\mathrm{H} / \mathrm{R}$ and applied $30 \mu \mathrm{M}$ pinocembrin to cells during the onset of reperfusion. In line with the effects of pinocembrin on the myocardial I/R injury, simulated I/R-reduced cell viability was significantly improved by pinocembrin (data not shown). Moreover, our data demonstrated that pinocembrin rescued cardiac troponin I (cTnI) release and LDH release post-simulated I/R in vitro, in both rat and mouse cardiomyocytes (fig 4 A-D).

Pinocembrin increases glycolysis in cardiomyocyte

During myocardial ischemia, enhanced glycolytic metabolism is essential for maintaining homeostasis of cardiomyocytes. In addition, previously studies reported that pinocembrin is involved in regulating glucose uptake in cancer cells (Liu et al., 2018). Subsequently, we explored the effects of pinocembrin on cellular bioenergetics with the Seahorse extracellular flux analyzer and performed glycolysis stress tests to measure glycolysis and glycolytic capacity both in intact rat and mouse cardiomyocytes. We observed that brief pretreatment with pinocembrin versus the control increased glycolysis by $21.4 \%$ (extracellular acidification rate $[\mathrm{ECAR}]$ ). Pinocembrin also increased glycolytic capacity in cardiomyocyte by $23.7 \%$ (fig 5 A-B).

To further explore the underlying mechanism of pinocembrin promoting myocardial glycolysis, mRNA expressions of a panel of glycolysis-related genes was determined with qRT-PCR. As shown in Fig 5C, pinocembrin significantly increased the expression of glycolysis-related genes, especially PFKFB3 gene.

3.5 PFKFB3 inhibition alters glycolysis and abolished pinocembrin-induced cardioprotection in cardiomyocytes

Next, we explored whether blockade of PFKFB3 inhibits cardiomyocyte glycolysis and impairs pinocembrinafforded cardioprotective effects. As shown in Fig 6 A-D, exposure of cardiomyocyte to $10 \mu \mathrm{M}$ PFKFB3 inhibitor, 3PO remarkably reversed pinocembrin-enhanced glycolysis. What's more, inhibition of PFKFB3 resulted in a significant increase of cTnI release and $\mathrm{LDH}$ release post-H/R in vitro (fig $6 \mathrm{E}-\mathrm{H}$ ), suggesting that disruption of glucose metabolism leads to impaired cardioprotection of pinocembrin during H/R.

3.6 PFKFB3 deficiency in normal mice using AAV9 abolished pinocembrin improved cardiac injury and dysfunction after $\mathrm{MI} / \mathrm{R}$.

Since that pinocembrin alleviated $\mathrm{H} / \mathrm{R}$-induced cardiomyocytes death by upregulating glycolysis via PFKFB3, we further evaluated the roles of PFKFB3 on myocardial I/R injury in vivo . To determine whether PFKFB3 regulation is directly involved in pinocembrin-related MI/R injury improvement, the WT mice were injected with AAV9 encoding PFKFB3 shRNA to knockdown endogenous PFKFB3. Intravenous injection of AAV9-shPFKFB3 on mice has successfully reduced protein level of PFKFB3 in heart (Supplementary fig 2). Subsequently, we exposed the mice to in situ myocardial ischemia-reperfusion to assess 
the functional role of myocardial-specific PFKFB3 in pinocembrin-afforded cardioprotection. We measured myocardial injury by infarct size area, serum cTnT levels and LDH activity. Exposure of mice infected with AAV9-shPFKFB3 to myocardial ischemia followed by reperfusion revealed larger infarct sizes and elevated levels of the myocardial necrosis marker troponin I and LDH release (fig 7 A-C). More importantly, delete myocardial PFKFB3 abolished pinocembrin-conferred protective effects on those indexes. Furthermore, the pinocembrin-improved EF and FS were also abolished by PFKFB3 deficiency with AAV9-shPFKFB3 (fig 7D). Collectively, these data support that pinocembrin confers cardioprotective effects by enhanced cardiomyocytes glycolysis through activation of PFKFB3.

\section{Discussion}

In the present studies, we address the functional roles of glycolysis in mediating cardioprotective responses of pinocembrin. We demonstrated that (i) pinocembrin delivered at the onset of reperfusion (postconditioning) significantly improved post-ischemic myocardial function and reduced infarct size after I/R ex vivo ; (ii) pinocembrin pretreatment significantly protected mouse hearts from acute myocardial I/R injury in vivo ; (iii) those protection is at least partially related to enhanced glycolysis by pinocembrin in the I/R cardiomyocytes; and (iv) the cardioprotective effects of pinocembrin are mediated by the activation of PFKFB3. These results extend previous findings indicating the cardioprotection of pinocembrin against $\mathrm{I} / \mathrm{R}$ injury and reveal the new mechanisms of pinocembrin in the cardioprotection.

It would be an attractive treatment principle to reduce the infarct size through pharmaceutical intervention to assist classic reperfusion intervention (Morel et al., 2012). Pinocembrin is a potential cardiovascular drug with potential neuroprotective effects on transient and long-term ischemic stroke in rats (Wu et al., 2013). Also, previous study indicated that administration of pinocembrin before myocardial ischemia improved LV function (Lungkaphin et al., 2015). Pharmacological postconditioning is easier to implement and has therapeutically potential in both clinical and experimental setting, which avoids the potential injury induced by ischemic conditioning, therefore has good clinical application prospects (Heusch, 2015). Therefore, our study aims to explore the cardioprotective effects of pinocembrin postconditioning. The ex vivo results showed that pinocembrin from 10 to $100 \mu \mathrm{M}$ delivered at the first 5 minutes of reperfusion remarkably improved post-ischemic myocardial function and attenuates cell death in a concentration-dependent manner. Furthermore, in vivo mouse myocardial I/R injury model was prepared and pinocembrin postconditioning was fulfilled by an intraperitoneal injection of pinocembrin $(5 \mathrm{mg} / \mathrm{kg}$ and $10 \mathrm{mg} / \mathrm{kg}$ body weight $) 5 \mathrm{~min}$ before reperfusion. Cardiac function, serum LDH activity and cTnT content, and infarct size were significantly improved with pinocembrin treatment. These data are consistent with the observation of cardioprotective effects of pinocembrin preconditioning others reported.

Various metabolic abnormalities occur during myocardial I/R, such as increased fatty acid oxidation and decreased glucose oxidation (Fillmore, Mori, \& Lopaschuk, 2014). This phenomenon is related to uncoupling of mitochondrial respiration, increased proton leakage, ROS formation and, more importantly, increased myocardial oxygen consumption (Boudina \& Abel, 2006). During myocardial I/R, the metabolic shift aimed at increasing glucose oxidation have proved to be beneficial. Although some therapeutic strategies have tried to reverse this metabolic imbalance, there is still no approved treatment regimen so far (Jaswal, Keung, Wang, Ussher, \& Lopaschuk, 2011; Kantor, Lucien, Kozak, \& Lopaschuk, 2000; Taniguchi et al., 2001). In addition, strategies aimed at increasing clinical glucose consumption have different results and have not yet reached routine clinical practice. Looking for drugs that can safely induce the transfer of cellular energy metabolism and a better understanding of the protective mechanisms of increased glucose oxidation may facilitate transfer to the clinic. Our results show that increasing glycolysis is responsible for pinocembrin induced protective effects. This is consistent with previous studies that metabolic shift towards increased glycolysis protects the heart from I/R injury (Nadtochiy et al., 2018). Moreover, PFKFB3 expression is notably upregulated during I/R, which directs cellular glucose metabolism from PPP to aerobic glycolysis (Li et al., 2019). Our data shown that PFKFB3 was significantly upregulated with pinocembrin treatment and specific inhibitor of PFKFB3 abolished pinocembrin-afforded protective effects in cardiomyocytes. Most importantly, knock 
down of PFKFB3 in myocardium using AAV9 reversed the cardioprotection of pinocembrin postconditioning. Previous studies have shown that the main reason responsible for the protection offered by the metabolic shift is the fact that glycolysis is able to produce two molecules of ATP without need for oxygen, so that uncoupling between mitochondrial full glucose oxidation and glycolysis leads to increased cardiac efficiency (Lopaschuk, 2017). However, potential protective mechanisms of the metabolic shift are largely unknown. Further studies are needed to investigate how pinocembrin regulate the glycolysis and search for its putative downstream targets.

In summary, our findings demonstrate that compound pinocembrin exhibits significant protective effects on cardiac I/R injury when delivered at the beginning of or before reperfusion in ex vivo rat andin vivo mouse models through enhancing glycolysis. Pinocembrin may be considered as an effective lead compound for large animal experiments, and is expected to be used in clinical research of acute myocardial infarction

Acknowledgements:

This work was supported by the Project of National Natural Science Foundation of China [grant numbers 81700354, 81970229].

Author contributions:

ZYJ and GXF performed and analysed the experiments. ZYJ wrote the first draft of the manuscript. WGQ participated in the analysis of the experiments. LJR revised the manuscript. ZYJ and LJR contributed to the experimental design and revised the manuscript.

Conflict of interest: No conflicts of interest.

Declaration of transparency and scientific rigour

This Declaration acknowledges that this paper adheres to the principles for transparent reporting and scientific rigour of preclinical research as stated in the BJP guidelines for Design \& Analysis, Immunoblotting and Immunochemistry, and Animal Experimentation, and as recommended by funding agencies, publishers and other organisations engaged with supporting research.

\section{References:}

Bice, J. S., \& Baxter, G. F. (2015). Postconditioning signalling in the heart: mechanisms and translatability. Br J Pharmacol, 172(8), 1933-1946. doi:10.1111/bph.12976 Boudina, S., \& Abel, E. D. (2006). Mitochondrial uncoupling: a key contributor to reduced cardiac efficiency in diabetes. Physiology (Bethesda), 21, 250-258. doi:10.1152/physiol.00008.2006 Fillmore, N., Mori, J., \& Lopaschuk, G. D. (2014). Mitochondrial fatty acid oxidation alterations in heart failure, ischaemic heart disease and diabetic cardiomyopathy. Br J Pharmacol, 171(8), 2080-2090. doi:10.1111/bph.12475 Gohil, V. M., Sheth, S. A., Nilsson, R., Wojtovich, A. P., Lee, J. H., Perocchi, F., . . . Mootha, V. K. (2010). Nutrient-sensitized screening for drugs that shift energy metabolism from mitochondrial respiration to glycolysis. Nat Biotechnol, 28(3), 249255. doi:10.1038/nbt.1606 Hanieh, H., Hairul Islam, V. I., Saravanan, S., Chellappandian, M., Ragul, K., Durga, A., . . . Thirugnanasambantham, K. (2017). Pinocembrin, a novel histidine decarboxylase inhibitor with anti-allergic potential in in vitro. Eur $J$ Pharmacol, 814, 178-186. doi:10.1016/j.ejphar.2017.08.012 He, X., Zeng, H., Chen, S. T., Roman, R. J., Aschner, J. L., Didion, S., \& Chen, J. X. (2017). Endothelial specific SIRT3 deletion impairs glycolysis and angiogenesis and causes diastolic dysfunction. $J$ Mol Cell Cardiol, 112, 104-113. doi:10.1016/j.yjmcc.2017.09.007 Heusch, G. (2015). Treatment of Myocardial Ischemia/Reperfusion Injury by Ischemic and Pharmacological Postconditioning. Compr Physiol, 5(3), 1123-1145. doi:10.1002/cphy.c140075 Hou, Z., Qin, X., Hu, Y., Zhang, X., Li, G., Wu, J., . . . Gao, F. (2019). Longterm Exercise-Derived Exosomal miR-342-5p: A Novel Exerkine for Cardioprotection. Circ Res, 124(9), 1386-1400. doi:10.1161/CIRCRESAHA.118.314635 Hunter, A. J., Hendrikse, A. S., \& Renan, M. J. (2007). Can radiation-induced apoptosis be modulated by inhibitors of energy metabolism? Int $J$ Radiat Biol, 83(2), 105-114. doi:10.1080/09553000601121157 Irvine, J. C., Cao, N., Gossain, S., Alexander, A. E., Love, J. E., Qin, C., . . . Ritchie, R. H. (2013). HNO/cGMP-dependent antihypertrophic actions 
of isopropylamine-NONOate in neonatal rat cardiomyocytes: potential therapeutic advantages of HNO over NO. Am J Physiol Heart Circ Physiol, 305(3), H365-377. doi:10.1152/ajpheart.00495.2012 Irvine, J. C., Ganthavee, V., Love, J. E., Alexander, A. E., Horowitz, J. D., Stasch, J. P., . . . Ritchie, R. H. (2012). The soluble guanylyl cyclase activator bay 58-2667 selectively limits cardiomyocyte hypertrophy. PLoS One, 7(11), e44481. doi:10.1371/journal.pone.0044481 Jaswal, J. S., Keung, W., Wang, W., Ussher, J. R., \& Lopaschuk, G. D. (2011). Targeting fatty acid and carbohydrate oxidation-a novel therapeutic intervention in the ischemic and failing heart.Biochim Biophys Acta, 1813(7), 1333-1350. doi:10.1016/j.bbamcr.2011.01.015 Jeong, D. W., Kim, T. S., Cho, I. T., \& Kim, I. Y. (2004). Modification of glycolysis affects cell sensitivity to apoptosis induced by oxidative stress and mediated by mitochondria. Biochem Biophys Res Commun, 313(4), 984-991. doi:10.1016/j.bbrc.2003.12.033 Kantor, P. F., Lucien, A., Kozak, R., \& Lopaschuk, G. D. (2000). The antianginal drug trimetazidine shifts cardiac energy metabolism from fatty acid oxidation to glucose oxidation by inhibiting mitochondrial long-chain 3-ketoacyl coenzyme A thiolase. Circ Res, 86(5), 580-588. doi:10.1161/01.res.86.5.580 Kishi, S., Campanholle, G., Gohil, V. M., Perocchi, F., Brooks, C. R., Morizane, R., . . . Bonventre, J. V. (2015). Meclizine Preconditioning Protects the Kidney Against Ischemia-Reperfusion Injury.EBioMedicine, 2(9), 1090-1101. doi:10.1016/j.ebiom.2015.07.035 Li, Z., Zhang, B., Yao, W., Zhang, C., Wan, L., \& Zhang, Y. (2019). APC-Cdh1 Regulates Neuronal Apoptosis Through Modulating Glycolysis and Pentose-Phosphate Pathway After Oxygen-Glucose Deprivation and Reperfusion. Cell Mol Neurobiol, 39(1), 123-135. doi:10.1007/s10571-018-0638-x Lionetti, V., Stanley, W. C., \& Recchia, F. A. (2011). Modulating fatty acid oxidation in heart failure. Cardiovasc Res, 90(2), 202-209. doi:10.1093/cvr/cvr038 Liu, Y., Liang, X., Zhang, G., Kong, L., Peng, W., \& Zhang, H. (2018). Galangin and Pinocembrin from Propolis Ameliorate Insulin Resistance in HepG2 Cells via Regulating Akt/mTOR Signaling. Evid Based Complement Alternat Med, 2018, 7971842. doi:10.1155/2018/7971842 Lopaschuk, G. D. (2017). Metabolic Modulators in Heart Disease: Past, Present, and Future. Can J Cardiol, 33(7), 838-849. doi:10.1016/j.cjca.2016.12.013 Lopaschuk, G. D., Ussher, J. R., Folmes, C. D., Jaswal, J. S., \& Stanley, W. C. (2010). Myocardial fatty acid metabolism in health and disease. Physiol Rev, 90(1), 207-258. doi:10.1152/physrev.00015.2009 Lungkaphin, A., Pongchaidecha, A., Palee, S., Arjinajarn, P., Pompimon, W., \& Chattipakorn, N. (2015). Pinocembrin reduces cardiac arrhythmia and infarct size in rats subjected to acute myocardial ischemia/reperfusion. Appl Physiol Nutr Metab, 40(10), 1031-1037. doi:10.1139/apnm2015-0108 Malmberg, K., Norhammar, A., Wedel, H., \& Ryden, L. (1999). Glycometabolic state at admission: important risk marker of mortality in conventionally treated patients with diabetes mellitus and acute myocardial infarction: long-term results from the Diabetes and Insulin-Glucose Infusion in Acute Myocardial Infarction (DIGAMI) study.Circulation, 99(20), 2626-2632. doi:10.1161/01.cir.99.20.2626 Matsushima, S., Kuroda, J., Ago, T., Zhai, P., Park, J. Y., Xie, L. H., . . . Sadoshima, J. (2013). Increased oxidative stress in the nucleus caused by Nox4 mediates oxidation of HDAC4 and cardiac hypertrophy. Circ Res, 112(4), 651-663. doi:10.1161/CIRCRESAHA.112.279760 Morel, O., Perret, T., Delarche, N., Labeque, J. N., Jouve, B., Elbaz, M., . . . Ovize, M. (2012). Pharmacological approaches to reperfusion therapy. Cardiovasc Res, 94(2), 246-252. doi:10.1093/cvr/cvs114 Nadtochiy, S. M., Wang, Y. T., Nehrke, K., Munger, J., \& Brookes, P. S. (2018). Cardioprotection by nicotinamide mononucleotide (NMN): Involvement of glycolysis and acidic pH. J Mol Cell Cardiol, 121, 155-162. doi:10.1016/j.yjmcc.2018.06.007 Qin, C. X., May, L. T., Li, R., Cao, N., Rosli, S., Deo, M., . . . Ritchie, R. H. (2017). Small-molecule-biased formyl peptide receptor agonist compound $17 \mathrm{~b}$ protects against myocardial ischaemia-reperfusion injury in mice. Nat Commun, 8, 14232. doi:10.1038/ncomms14232 Saad, M. A., Abdel Salam, R. M., Kenawy, S. A., \& Attia, A. S. (2015). Pinocembrin attenuates hippocampal inflammation, oxidative perturbations and apoptosis in a rat model of global cerebral ischemia reperfusion.Pharmacol Rep, 67(1), 115-122. doi:10.1016/j.pharep.2014.08.014 Shi, L. L., Chen, B. N., Gao, M., Zhang, H. A., Li, Y. J., Wang, L., \& Du, G. H. (2011). The characteristics of therapeutic effect of pinocembrin in transient global brain ischemia/reperfusion rats.Life Sci, 88(11-12), 521-528. doi:10.1016/j.lfs.2011.01.011 Somanathan, S., Jacobs, F., Wang, Q., Hanlon, A. L., Wilson, J. M., \& Rader, D. J. (2014). AAV vectors expressing LDLR gain-of-function variants demonstrate increased efficacy in mouse models of familial hypercholesterolemia. Circ Res, 115(6), 591-599. doi:10.1161/CIRCRESAHA.115.304008 Taniguchi, M., Wilson, C., Hunter, C. A., Pehowich, D. J., Clanachan, A. S., \& Lopaschuk, G. D. (2001). Dichloroacetate improves cardiac efficiency after ischemia independent of changes in mitochondrial pro- 
ton leak. Am J Physiol Heart Circ Physiol, 280(4), H1762-1769. doi:10.1152/ajpheart.2001.280.4.H1762 Tao, J., Shen, C., Sun, Y., Chen, W., \& Yan, G. (2018). Neuroprotective effects of pinocembrin on ischemia/reperfusion-induced brain injury by inhibiting autophagy. Biomed Pharmacother, 106, 1003-1010. doi:10.1016/j.biopha.2018.07.026 Vaughn, A. E., \& Deshmukh, M. (2008). Glucose metabolism inhibits apoptosis in neurons and cancer cells by redox inactivation of cytochrome c. Nat Cell Biol, 10(12), 14771483. doi:10.1038/ncb1807 Wu, C. X., Liu, R., Gao, M., Zhao, G., Wu, S., Wu, C. F., \& Du, G. H. (2013). Pinocembrin protects brain against ischemia/reperfusion injury by attenuating endoplasmic reticulum stress induced apoptosis.Neurosci Lett, 546, 57-62. doi:10.1016/j.neulet.2013.04.060 Xie, C., Zhang, Y. P., Song, L., Luo, J., Qi, W., Hu, J., . . . Yan, Y. (2016). Genome editing with CRISPR/Cas9 in postnatal mice corrects PRKAG2 cardiac syndrome. Cell Res, 26(10), 1099-1111. doi:10.1038/cr.2016.101 Zhang, P., Xu, J., Hu, W., Yu, D., \& Bai, X. (2018). Effects of Pinocembrin Pretreatment on Connexin 43 (Cx43) Protein Expression After Rat Myocardial Ischemia-Reperfusion and Cardiac Arrhythmia. Med Sci Monit, 24, 50085014. doi:10.12659/MSM.909162 Zheng, Y., Gu, S., Li, X., Tan, J., Liu, S., Jiang, Y., . . . Yang, H. T. (2017). Berbamine postconditioning protects the heart from ischemia/reperfusion injury through modulation of autophagy. Cell Death Dis, 8(2), e2577. doi:10.1038/cddis.2017.7

\section{Figure legends}

Figure 1. Experimental protocols to investigate the effects of pinocembrin on MI/R. (A) Protocol of I/R ex vivo study (1). (B) Protocol of MI/R in vivo study.

Figure 2. Compound pinocembrin significantly improves cardiac contractile recovery, reduces infarct size and and LDH leakage after ischemia-reperfusion (I/R) ex vivo . A-D. Time course of LVDP (A), LVEDP $(B)$, and $+\mathrm{dP} / \mathrm{dtmax}(\mathrm{C})$ and $-\mathrm{dP} / \mathrm{dtmax}(\mathrm{D}) ; \mathrm{n}=10$ each. E. Effect of pinocembrin on the LDH activity in the coronary effluent; $\mathrm{n}=10$ each. $\mathrm{F}$. Representative images and analysis of the infarct size in isolated $\mathrm{I} / \mathrm{R}(30 \mathrm{~min} / 2 \mathrm{~h})$ hearts; $\mathrm{n}=7$ in control group; $\mathrm{n}=10$ in pinocembrin group. Data represent the mean \pm SEM. ${ }^{*} P<0.05$ versus I/R control group.

Figure 3. Pinocembrin protects from myocardial I/R (MI/R) injury in vivo in C57/BL6 mice. Wild-type (WT) mice were subjected to 30 minutes of the left anterior descending coronary artery (LAD) ligation, followed by $24 \mathrm{~h}$ of reperfusion, vehicle or pinocembrin intravenously ( 5 and $10 \mathrm{mg} / \mathrm{kg}$ ) $5 \mathrm{~min}$ before reperfusion. A. Representative 2,3,5-triphenyltetra-zolium chloride (TTC)-stained LV transverse slices (scale bar,1 $\mathrm{mm}$ ). Areas stained dark blue, white and red represented non-risk, infarcted and ischaemic but non-infarcted zones, respectively. B. Analysis of AAR (calculated as total infarcted plus ischaemic but non-infarcted zones, $\%$ total LV). C. Analysis of Myocardial infarct size (\%AAR). D-E, Plasma LDH activity and cTnI levels. F-G, Representative images and left ventricular ejection fraction (EF\%) and fractional shortening (FS\%) by echocardiography. $\mathrm{n}=9$ each. Data represent the mean \pm SEM. ${ }^{*} P<0.05$ versus sham group. ${ }^{*} P<0.05$ versus I/R control group.

Figure 4. Protective actions of pinocembrin against cardiomyocyte injury responses in vitro. A. Effect of pinocembrin $(30 \mu \mathrm{M}$, added during reperfusion) on rat cardiomyocyte $\mathrm{cTnI}$ release subsequent to $\mathrm{H} / \mathrm{R}$. B. Effect of pinocembrin on rat cardiomyocyte LDH release subsequent to H/R. C. Effect of pinocembrin on mouse cardiomyocyte cTnI release subsequent to H/R. D. Effect of pinocembrin on mouse cardiomyocyte $\mathrm{LDH}$ release subsequent to $\mathrm{H} / \mathrm{R}$. $\mathrm{n}=5$ each. Data represent the mean $\pm \mathrm{SEM} .{ }^{*} P<0.05$ versus normoxia with vehicle group. ${ }^{\#} P<0.05$ versus $\mathrm{H} / \mathrm{R}$ with vehicle group.

Figure 5. Pinocembrin increases glycolysis in primary cardiomyocytes. A. Seahorse XFe24 extracellular flux analyzer traces of a glycolysis stress test in rat cardiomyocytes measured as the ECAR by sequential injection of $20 \mathrm{mM}$ glucose, $1 \mu \mathrm{M}$ oligomycin, and $100 \mathrm{mM}$ 2-deoxy-d-glucose (2-DG). B. Quantification of glycolysis, glycolytic capacity, and glycolytic reserves in rat cardiomyocytes pretreated with pinocembrin or control. C. Seahorse XFe24 extracellular flux analyzer traces of a glycolysis stress test in mouse cardiomyocytes measured as the ECAR by sequential injection of $20 \mathrm{mM}$ glucose, $1 \mu \mathrm{M}$ oligomycin, and $100 \mathrm{mM} 2$-DG. D. Quantification of glycolysis, glycolytic capacity, and glycolytic reserves in mouse cardiomyocytes pretreated with pinocembrin or control. E. Mouse cardiomyocytes were treatment with pinocembrin for $24 \mathrm{~h}$, and 
then the glycolysis-related genes were detected by qRT-PCR. $\mathrm{n}=3$ /treatment in all Seahorse assays, each in triplicate. Data represent the mean \pm SEM. ${ }^{*} P<0.05$, significantly different as indicated. NS, no significant difference as indicated.

Figure 6. Pinocembrin protect cardiomyocytes from simulated I/R injury via enhancing glycolysis. A-D Treatment with PFKFB3 inhibitor, 3PO, results in decline of glycolysis and glycolytic capacity in mouse and rat cardiomyocytes. $n=3 /$ treatment in all Seahorse assays, each in triplicate. E-F. Effect of PFKFB3 inhibitor, 3PO, on mouse and rat cardiomyocyte $\mathrm{cTnI}$ release subsequent to H/R. n=5 each. G-H. Effect of PFKFB3 inhibitor, 3PO, on mouse and rat cardiomyocyte LDH release subsequent to $\mathrm{H} / \mathrm{R}$. $\mathrm{n}=5$ each. Data represent the mean \pm SEM. ${ }^{*} P<0.05$ versus $H / R$ with vehicle group. ${ }^{\#} P<0.05$ versus $H / R$ with $P B$ group.

Figure 7. Knock down of PFKFB3 in myocardium using AAV9 impairs pinocembrin induced cardioprotection after MI/R. A. Representative infarct staining from AAV-shNC or AAV-shPFKFB3 mice treated with vehicle or pinocembrin. B-C AAR and infarct sizes from AAV-shNC or AAV-shPFKFB3 mice treated with vehicle or pinocembrin. D-E. Plasma LDH activity and cTnI levels. F. Representative echocardiographic images, assessment of LV EF and FS by echocardiography from AAV-shNC or AAV-shPFKFB3 mice treated with vehicle or pinocembrin. $\mathrm{n}=8$ each. Data represent the mean \pm SEM. ${ }^{*} P<0.05$, significantly different as indicated.

\section{Figure 1}

A

Time of ex vivo study
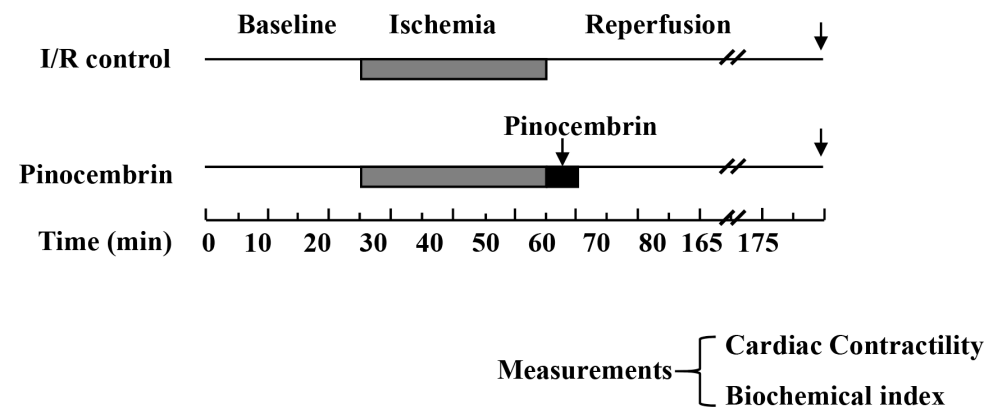

B

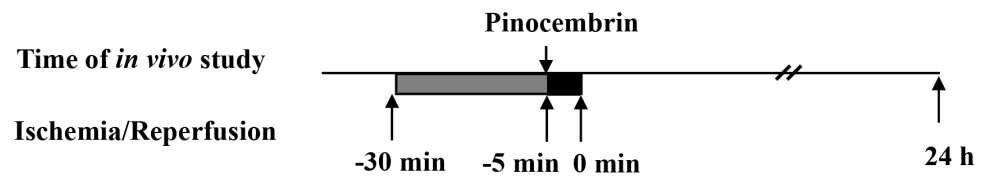

Measurements $\left\{\begin{array}{l}\text { Cardiac function } \\ \text { Infarc Size } \\ \text { Biochemical index }\end{array}\right.$ 
Figure 2

A

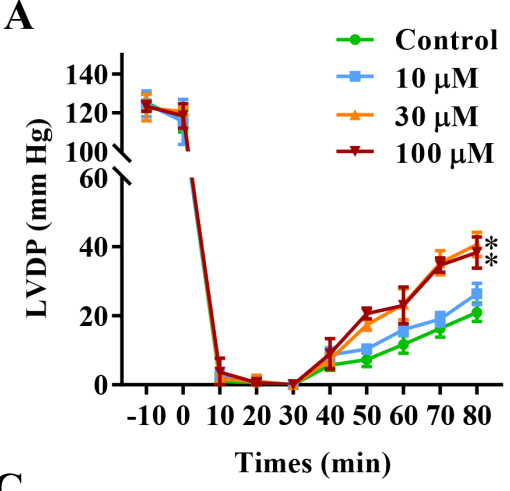

C

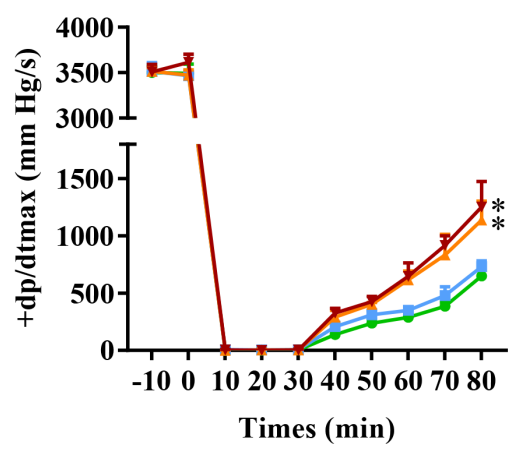

E

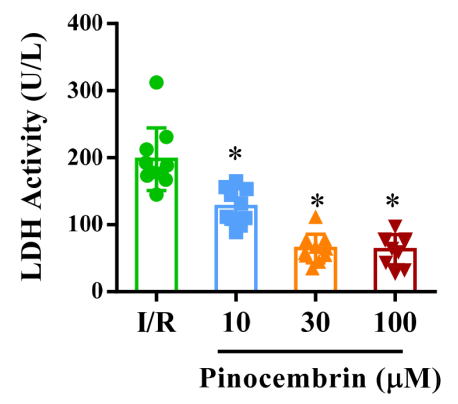

B

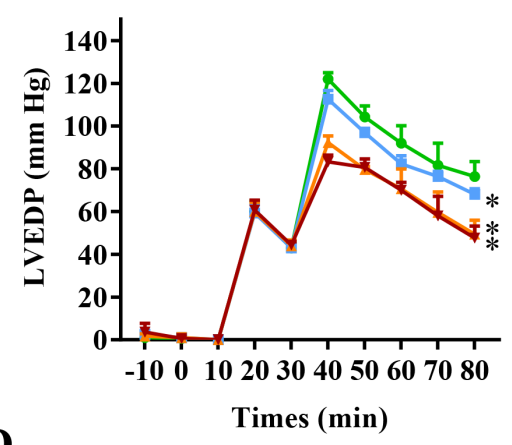

D

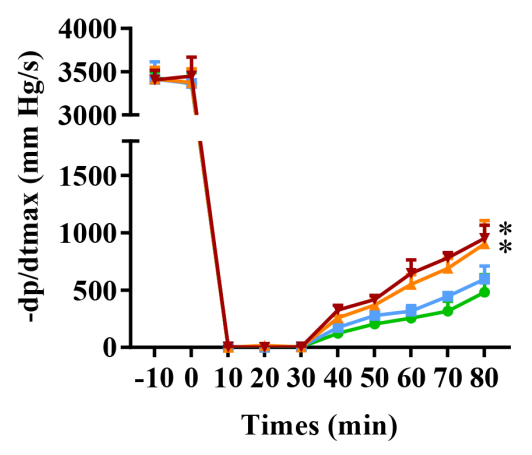

F
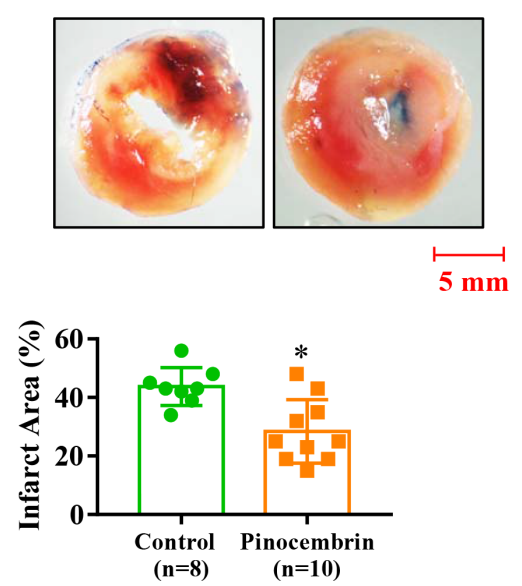
Figure 3

A

I/R+Pinocembrin (mg/kg)

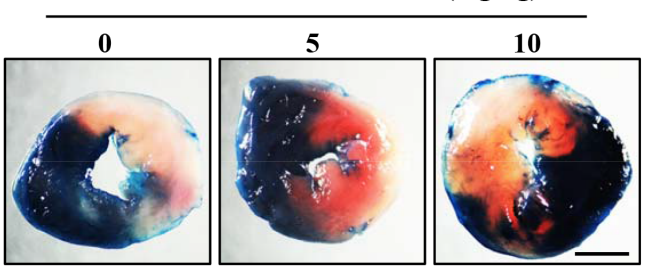

B

C

$1 \mathrm{~mm}$
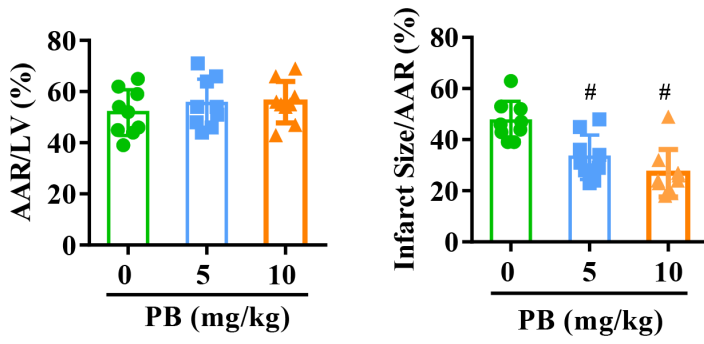

D

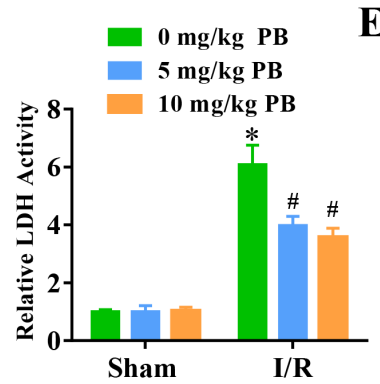

E

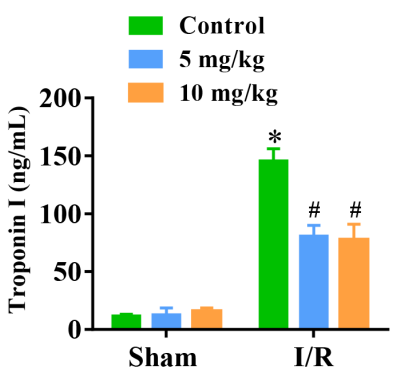

F
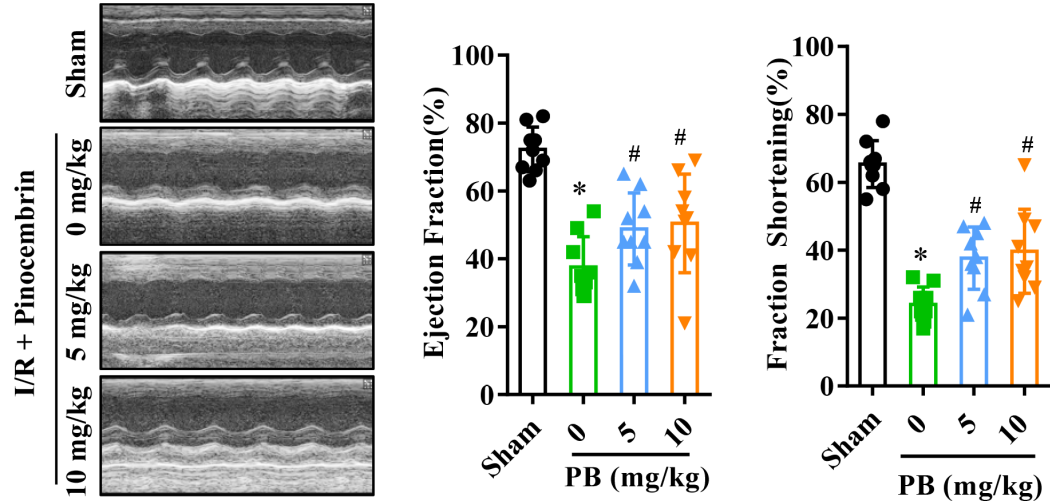
Figure 4

A

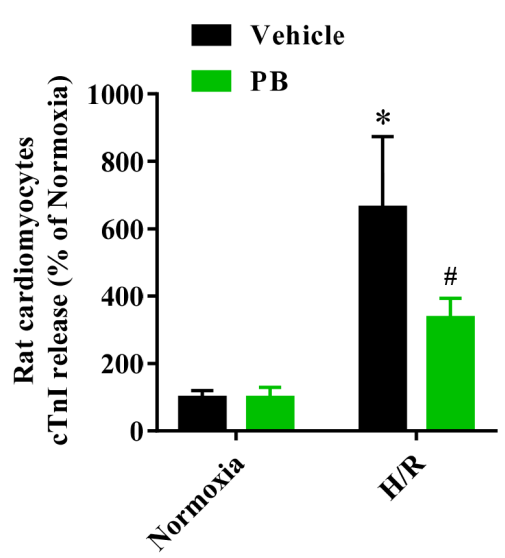

C

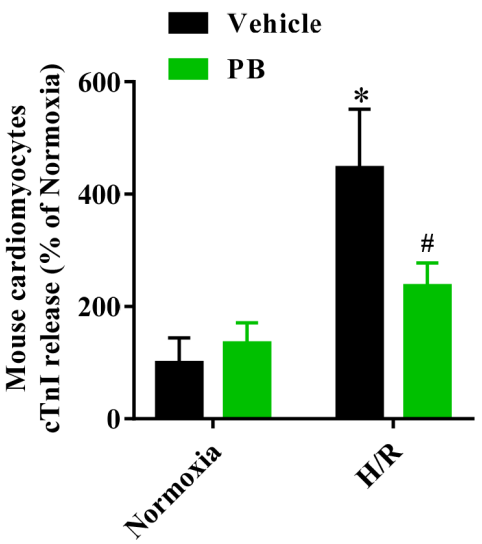

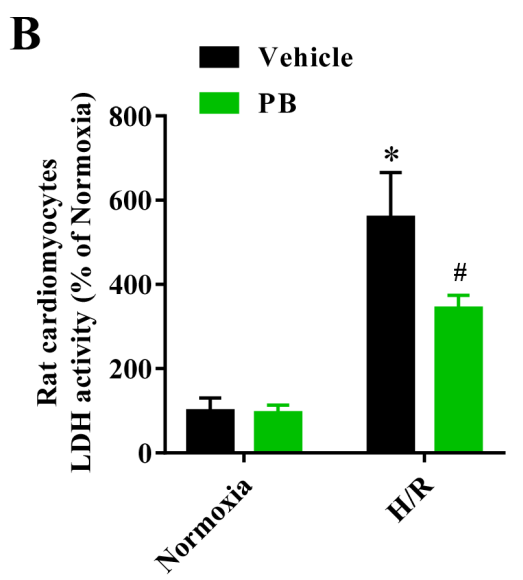

D

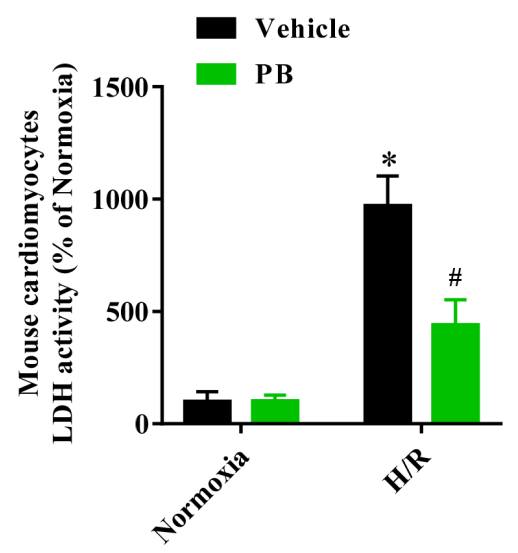


Figure 5

A
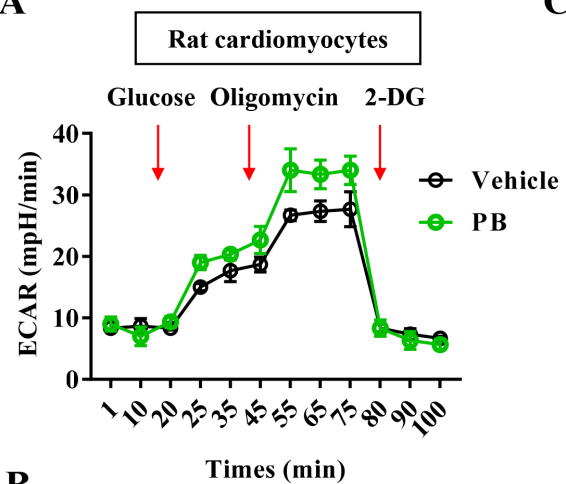

B

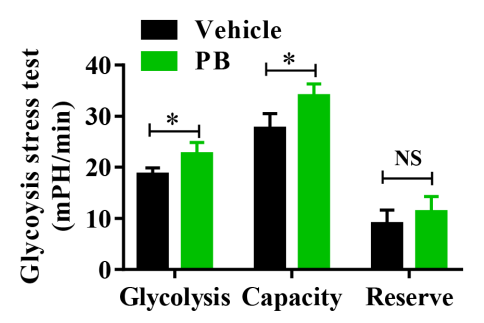

E

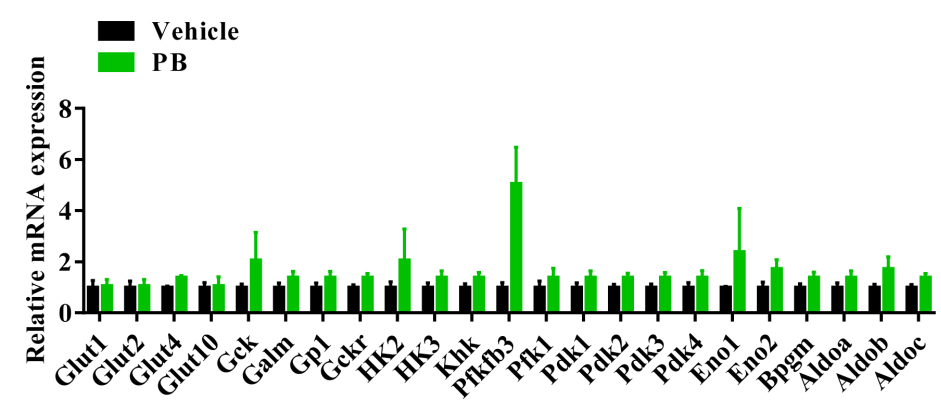

C

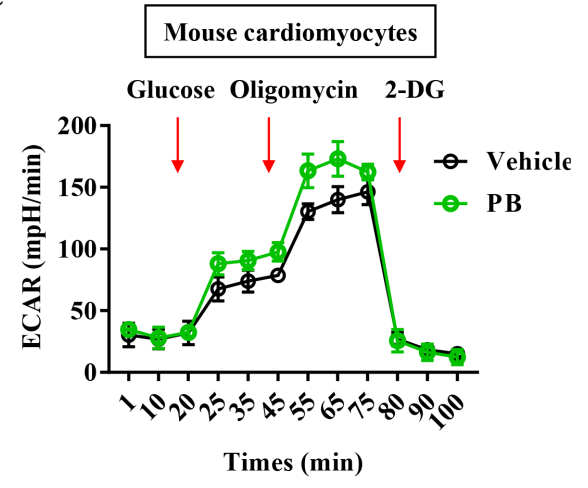

D

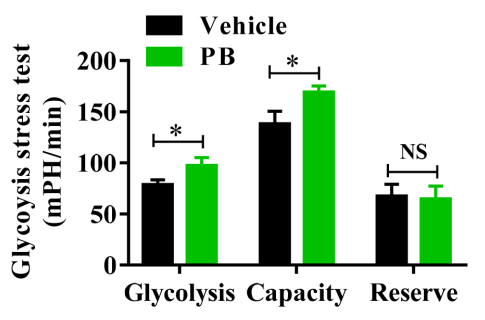


Figure 6

A

C
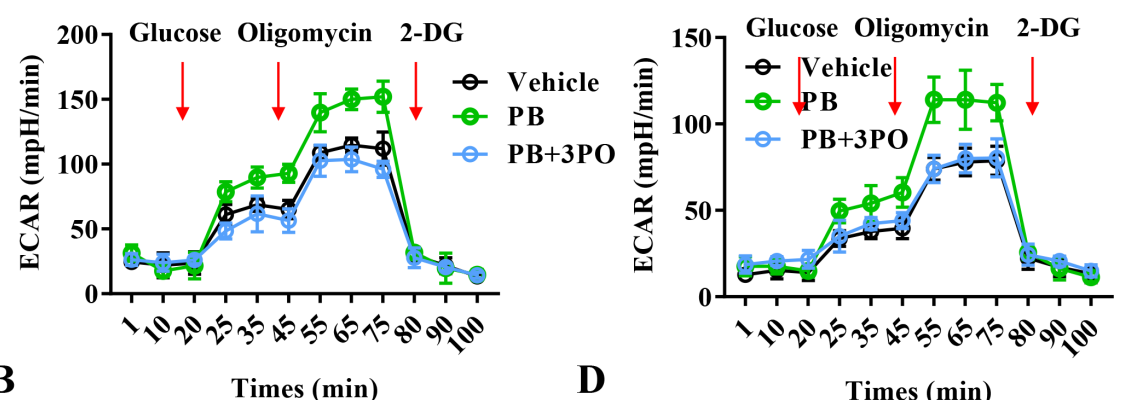

B

Times (min)

D
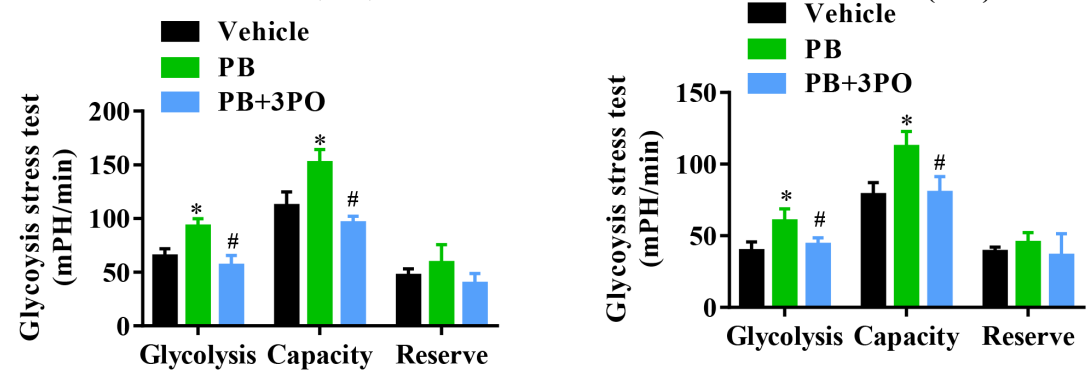

E

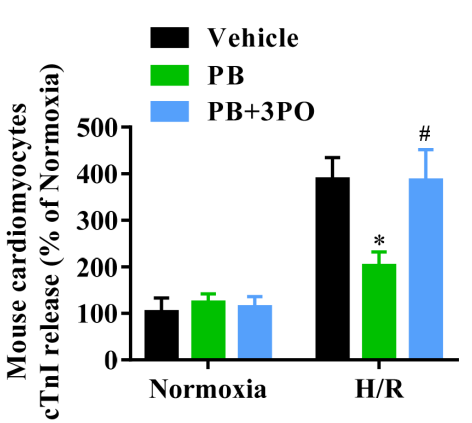

F

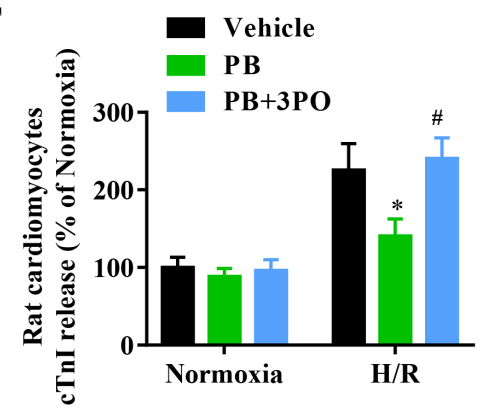

G

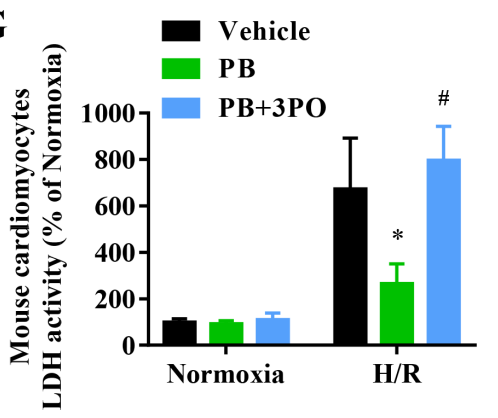

H

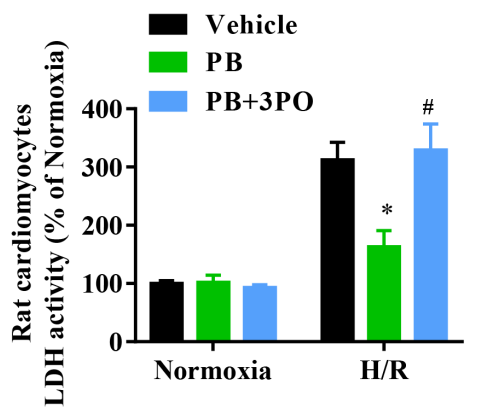


Figure 7

A

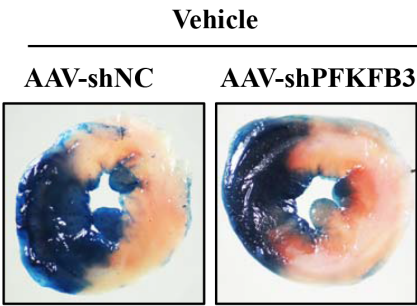

B
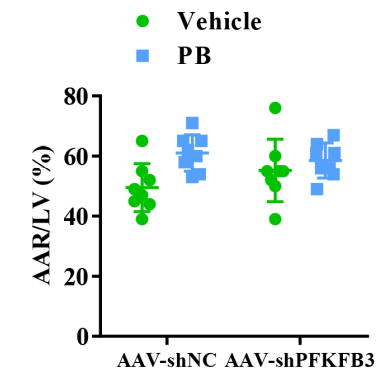

D

- Vehicle

- PB

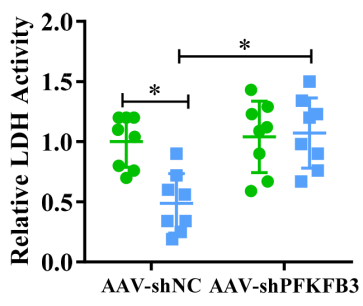

PB

AAV-shPFKFB3

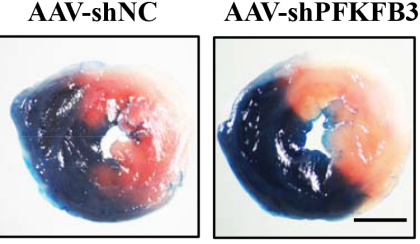

C

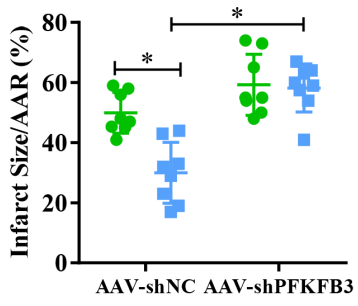

E

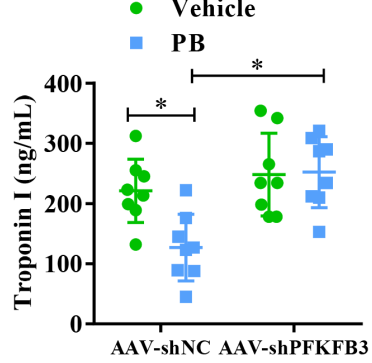

F

\begin{tabular}{c|c}
\hline \multicolumn{2}{c}{ Vehicle } \\
\cline { 1 - 1 } & \\
\hline
\end{tabular}

\begin{tabular}{|c|c|}
\hline PB & \\
\hline & \\
\hline & \\
\hline
\end{tabular}
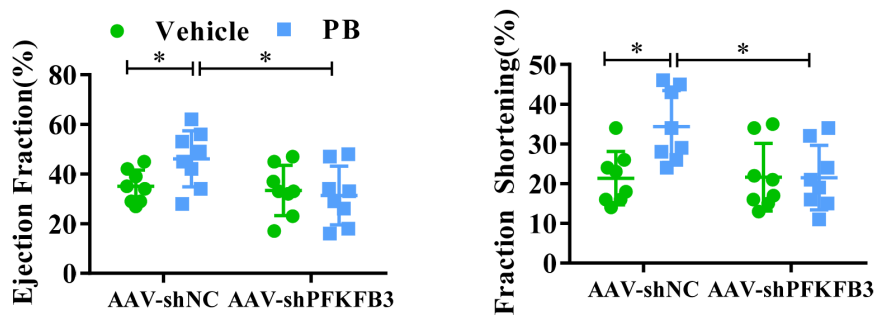